\title{
Algorithms of ionospheric anomalies detection in "Aurora" system of operational data analysis
}

\author{
Yuryi Polozov, and Nadezhda Fetisova \\ Institute of Cosmophysical Research and Radio Wave Propagation FEB RAS, 684034, Kamchatsky \\ krai, Paratunka, Russian Federation
}

\begin{abstract}
Algorithms for ionospheric data processing are presented in the paper. The algorithms are implemented in the real-time mode of ionospheric parameter analysis. They are a component of "Aurora" software system for geophysical data analysis. The algorithms allow us to estimate the state of the ionosphere in the region of Kamchatka Peninsula and to detect ionospheric anomalies. Assessment of the algorithms efficiency has shown that it is possible to use them to detect ionospheric anomalies that may occur on the eve of magnetic storms. The research is supported by the Russian Science Foundation Grant (Project No. 14-1100194).
\end{abstract}

\section{Introduction}

The paper is aimed at constructing the automatic algorithms for processing and analysis of ionospheric parameter time series and software solutions on their basis. We present the algorithms which are based on the methods for modelling and analysis of natural time series of complicated structure [1-4]. Ionospheric parameters have complicated structure, include diurnal and seasonal components and depend on solar activity, geographic coordinates and geomagnetic conditions [5-7]. Ionospheric disturbances occur during increased solar (flares, coronal ejections) and geomagnetic activities. They manifest in the recorded parameters as anomalous features of different structure and duration. Powerful and long disturbances in the ionosphere are the cause of operational disorder in ground and space technical means $[5,6]$, thus, the timely detection of them is an important task [5-8]. Disturbances are characterized by significant changes of electron concentration in the ionosphere, they may have positive and negative phases, different intensity and duration [5, 7].

It is difficult to apply the traditional analysis methods (regression, smoothing, spectral, ARIMA [9] and so on) to detect and to estimate anomalous changes in the ionospheric parameters owing to the nonstationary character of ionospheric dynamics changes. In this paper we apply a generalized multicomponent model (GMCM) [2, 10], which allows us to model ionospheric parameter time series. We can describe ionospheric parameter regular variations and anomalous changes by the GMCM. The basis of GMCM is a complex approach using the wavelet transform methods and ARIMA model. For the first time, this approach was proposed in the paper [2]. Numerical realization of the model [10] allowed representing it in the form of software solutions for ionospheric parameter analysis in real- 
time mode. To identify and to estimate the detected anomalies, the authors of the paper suggested applying continuous wavelet transform (CWT) $[2,11,12]$. Application of CWT and adaptive threshold functions for ionospheric parameter analysis is based on the papers $[2,12,13]$. The proposed approach was realized in the form of numerical algorithms and represented in software solutions. Another approach for ionospheric parameter modeling is based on the combination of wavelet transform and neural networks. The results of its application show the possibility of detection of ionospheric disturbances [14-16]. The neural networks perform their operation with ionospheric data after their preliminary wavelet processing. In this case, the time series noise component is suppressed that allows increasing the efficiency of neural network operation during anomaly detection $[14,15]$. The approach is implemented in software solutions which process data in real-time mode. The described approaches are used for the analysis of ionospheric F2 layer (foF2) critical frequency data (Paratunka station).

\section{2 lonospheric time series analysis methods realized in real-time mode}

\subsection{Modeling of ionospheric parameters by GMCM}

Ionospheric parameter time series has a regula and an anomalous components and can be represented in the form of a generalized multicomponent model (GMCM) [10]:

$$
\mathrm{f}(\mathrm{t})=\mathrm{R}(\mathrm{t})+\mathrm{U}(\mathrm{t})+\mathrm{e}(\mathrm{t})=\sum_{\mu=\overline{1, T}} \alpha^{\mu}(\mathrm{t})+\sum_{\eta} \beta_{\text {dist }}^{\eta}(\mathrm{t})+\mathrm{e}(\mathrm{t})
$$

where $\mathrm{R}(\mathrm{t})=\sum_{\mu=\overline{1, T}} \alpha^{\mu}(\mathrm{t})(\mu=\overline{1, \mathrm{~T}}$ is the component number) is a recurrent component describing ionospheric parameter regular variations (during the periods of absence of ionospheric disturbances; $\mathrm{U}(\mathrm{t})=\sum_{\eta} \beta_{\text {dist }}^{\eta}(\mathrm{t})$ is the irregular (anomalous) component; $\mathrm{e}(\mathrm{t})$ is a random component.

It was shown in the paper [10] that the recurrent component can be represented in the form

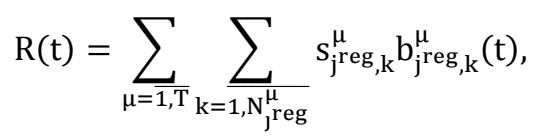

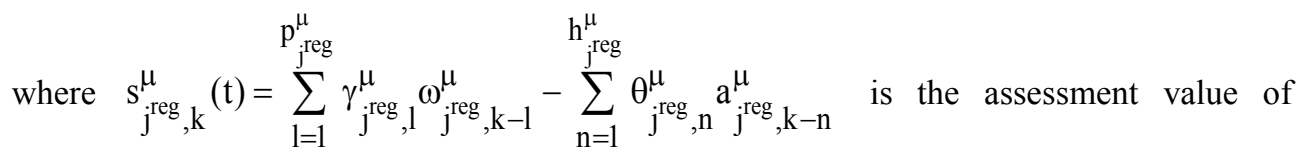
regular $\mu$-th component, $\mathrm{p}_{\mathrm{j} \text { reg }}^{\mu}, \gamma_{\mathrm{j} \text {, reg }, 1}^{\mu}$ are the order and parameters of $\mu$-th component autoregression, $\mathrm{h}_{\mathrm{j} \text { reg }}^{\mu}, \theta_{\mathrm{j}}^{\mu}{ }_{\text {reg }, \mathrm{n}}^{\mathrm{are}}$ the order and parameters of a moving average of $\mu$-th component, $\omega_{\mathrm{j}^{\text {reg }}, \mathrm{k}}^{\mu}=\nabla^{\nu^{\mu}} \delta_{\mathrm{j}^{\mathrm{reg}}, \mathrm{k}}^{\mu}, \nu^{\mu}$ is the difference order of $\mu$-th component,

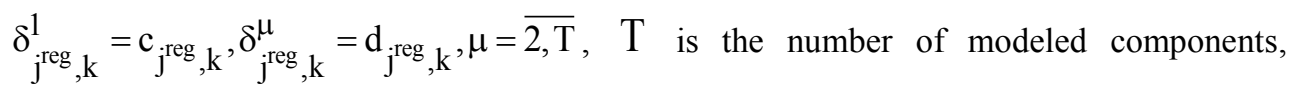
$\mathrm{a}_{\mathrm{j}}{ }_{\text {reg }}$ are residual errors of $\mu$-th component model, $\mathrm{N}_{\mathrm{j}}^{\mu}$ reg is the length of $\mu$-th 
component, $b_{\mathrm{j}^{\text {reg }}, \mathrm{k}}^{1}=\phi_{\mathrm{j}{ }_{\mathrm{reg}}, \mathrm{k}}$ is the scaling function, $\mathrm{b}_{\mathrm{j}^{\mathrm{reg}}{ }_{, \mathrm{k}}}^{\mu}=\Psi_{\mathrm{j}^{\mathrm{reg}}, \mathrm{k}}, \mu=\overline{2, \mathrm{~T}}$ is the wavelet basis of $\mu$-th component.

\subsection{Identification and detailed analysis of anomalies}

As long as the components $\beta_{\text {dist }}^{\eta}(t)$ of irregular component $U(t)$ of model (1) have local structure, the most effective way of their description is the application of nonlinear adaptive approximating scheme and the following relation is fair [12]:

$$
U(t)=\sum_{\eta} \beta_{\mathrm{dist}}^{\eta}(t)=\sum_{(\eta, n) \in P_{M}} d_{\eta, n} \Psi_{\eta, n}(t)+\sum_{(\eta, n) \notin P_{M}} d_{\eta, n} \Psi_{\eta, n}(t)=U_{M}(t)+e(t),
$$

where $U_{M}(t)=\sum_{(\eta, n) \in P_{M}}\left\langle f, \Psi_{\eta, n}\right\rangle \Psi_{\eta, n}(t)$ is the projection of $U(t)$ on $M$ vectors, the indices of which are contained in some set $P_{M}$; the component $e(t)=\sum_{(\eta, n) \notin P_{M}}\left\langle f, \Psi_{\eta, n}\right\rangle \Psi_{\eta, n}(t)$ is the sequence of noise factor effect (we assume this component to be uncorrelated and additive).

Identification of the component $U_{M}(t)$ can be based on the application of threshold functions [11]

$$
U_{M}(t)=\left\{\begin{array}{l}
\sum_{\eta, n} d_{\eta, n} \Psi_{\eta, n}(t), \text { если }\left|d_{\eta, n}\right| \geq T_{\eta} \\
0, \text { если }\left|d_{\eta, n}\right|<T_{\eta}
\end{array}\right.
$$

Taking into account the significant nonstationarity of the modeled time series, adaptive thresholds $T_{\eta}=T_{\eta}^{a d}$ and coefficients $\left\{d_{\eta, n}\right\}_{(\eta, m) \in P_{M}}$ were introduced in the paper in the relation (4) to be equal $[12,14]$

$$
d_{\eta, n}= \begin{cases}d_{\eta, n}^{+}, & \text {if }\left(d_{\eta, n}-d_{\eta, n}^{\text {med }}\right) \geq T_{\eta}^{a d} \\ d_{\eta, n}^{-}, & \text {if }\left(d_{\eta, n}-d_{\eta, n}^{\text {med }}\right) \leq-T_{\eta}^{a d}\end{cases}
$$

where $T_{\eta}^{a d}=V^{*} S t_{\eta}$, the value $S t_{\eta}=\sqrt{\frac{1}{\Phi-1} \sum_{n=1}^{\Phi}\left(d_{\eta, n}-\overline{d_{\eta, n}}\right)^{2}}, \overline{d_{\eta, n}}$ and $d_{\eta, n}^{\text {med }}$ is the average and the median, respectively. Taking into account the ionospheric data diurnal variation, they are calculated within the moving time window of the length $\Phi$.

As long as the wavelet coefficient $\left|d_{\eta, n}\right|$ amplitude characterizes the anomalous feature anmplitude on scale $\eta$ (see[11]), it is logical to assess it as a measure of anomaly intensity of scale $\eta$. We determine the anomaly intensity at a time instant $t=n$ as

$$
I_{n}=\sum_{\eta}\left|d_{\eta, n}\right|
$$

\subsection{Modeling of ionospheric parameters by neural network}

Following the papers $[14,15]$, ionospheric parameter time variation modeling is carried out on the basis of the operations below. 
Based on multiresolution wavelet decompositions, we represent the time series $f_{0}(t)$ in the form of linear combination of different-scale components: smoothed one $f\left[2^{-m} t\right]$ of scale $\mathrm{m}$ and detailing ones $g\left[2^{j} t\right]$ of scales $j=\overline{-1,-m}[11]$ :

$$
f_{0}(t)=\sum_{j=-1}^{-m} g\left[2^{j} t\right]+f\left[2^{-m} t\right]
$$

where the different-scale detailing components $g\left[2^{j} t\right]=\sum_{k} d_{j, k} \Psi_{j, k}(t), d_{j, k}=\left\langle f, \Psi_{j, k}\right\rangle$, $\Psi$ is the wavelet basis, $j$ is the resolution; approximating component $f\left[2^{-m} t\right]=\sum_{k} c_{-m, k} \varphi_{-m, k}(t), c_{-m, k}=\left\langle f, \varphi_{-m, k}\right\rangle, \varphi$ is the smoothing scaling function. The suffix 0 corresponds to the data initial resolution.

Applying the inverse wavelet transform [11], the initial resolution $j=0$ is restored for the component $f\left[2^{-3} t\right]=\sum_{k} c_{-3, k} \varphi_{-3, k}(t)$ :

$$
f_{0}^{a p}(t)=\sum_{k} c_{0, k} \varphi_{0, k}(t)
$$

where $c_{0 k}=\left\langle f_{0}^{a p}, \varphi_{\text {to }}\right\rangle, \varphi_{0, k}(t)=\varphi(t-k)$. Based on the preliminary trained neural networks $(\mathrm{NN})$, the component $f_{0}^{a p}(t)$ is modeled.

NN were trained on foF2 data for the period 1968-2010 (Paratunka st.). To improve the quality of approximation for training, we used the periods without strong magnetic storms and high seismic activity in Kamchatka.

The constructed NN predict the data $[14,15]$ :

$$
c_{-3, k+1}(t)=\phi_{3}\left(\sum_{i} \omega_{i j} \phi_{2}\left(\sum_{q} \omega_{q i} \phi_{1}\left(\sum_{k} \omega_{k q} c_{-3, k}(t)\right)\right),\right.
$$

where $\omega_{k q}$ are the weight coefficients of neuron $q$ of network input layer, are the weight coefficients of neuron $i$ of network hidden layer, $\omega_{i j}$ are the weight coefficients of neuron $j$ of output layer, $\phi_{1}(z)=\phi_{2}(z)=\frac{1}{1+\exp (-z)}, \phi_{3}(z)=a * z+b$.

A trained NN makes it possible to reconstruct the characteristic foF2 time variation and the procedure of anomalous changes detection can be based on the analysis of $\mathrm{NN}$ errors when the characteristic variation is disturbed $[14,15]$.

\section{Results of application of numerical algorithms in software tools}

The approaches described in Section 2 were represented in the form of numerical algorithms $[10,14,15]$ and implemented in the form of software block for data processing in real-time mode. The block is included into the structure of «Auroroa» software system for data analysis (http://aurorasa.ikir.ru:8580). The results of ionospheric data processing by software solutions for Paratunka station (IKIR FEB RAS) are illustrated in Fig. 1-3. We 
processed the data which were obtained during the magnetic storm on November 24, 2016. It began at 00:00 UT. Fig. 1 shows the results for the period which includes the magnetic storm. Based on the approach of Section 2.2, we detected positive and negative anomalies (red and blue, respectively) in the ionosphere (Fig. 1 b,c), which characterize electron concentration change. Analysis of Fig. 1 b,c shows a long positive anomaly (electron concentration increase) before the magnetic storm. A negative anomaly was detected in the ionosphere during the magnetic storm recovery phase. Modeling of foF2 based on the approaches from Sections 2.1 and 2.3 (Fig. $1 \mathrm{~d}$, e) shows model error increase during the negative anomaly that indicates the disturbance of the ionospheric process characteristic variation.

Fig. 2, 3 illustrate the method applicability for real-time detection of anomalies in the ionosphere. Fig. 2 shows the period of data processing till 14:00 UT on 2016.11.23. It is clear from the graphs (Fig. 2 b, c), a positive anomaly begins to form before the storm. In Fig. 3, the processing is carried out till 17:00 UT on 2016.11.23. A positive anomaly is formed before the magnetic storm and its intensity begins to grow (Fig. 3 b, c). The processing results show the possibility of timely detection of ionospheric anomalies and assessment of their occurrence dynamics.
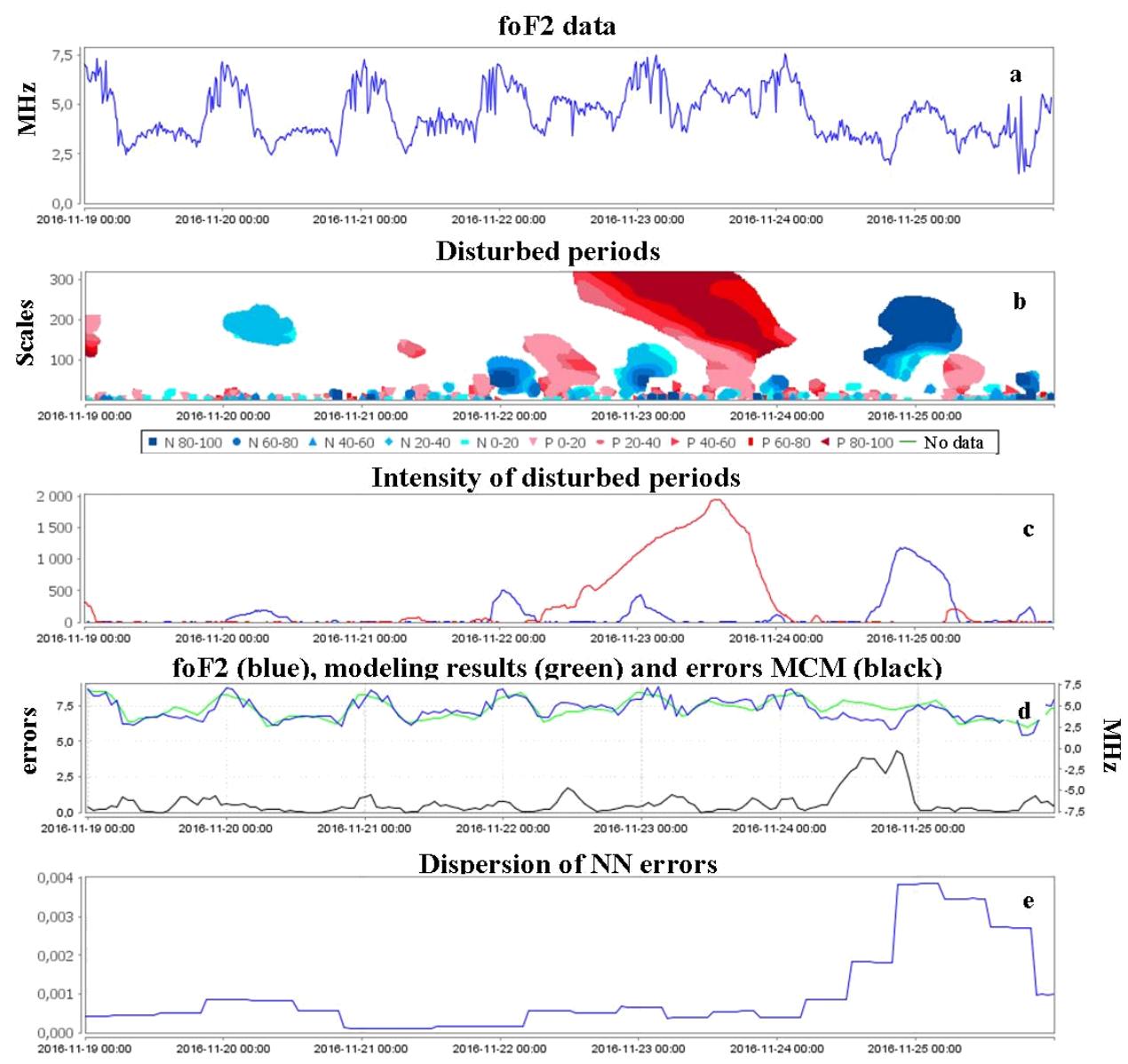

Fig. 1. Results of foF2 data processing for the period 2016.11.19-2016.11.25. 

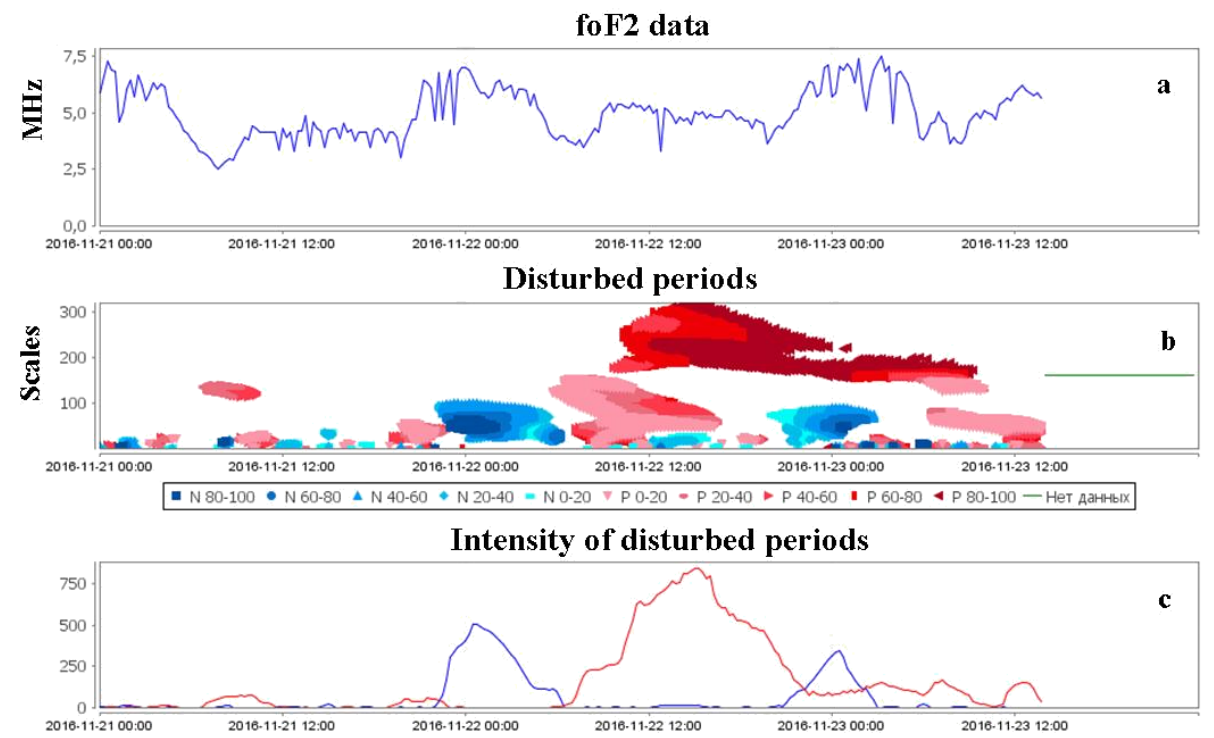

Fig. 2. Results of foF2 data processing for the period 2016.11.21 - 2016.11.23. The foF2 data were processes till 14:00 UT on 2016.11.23.

\section{foF2 data}
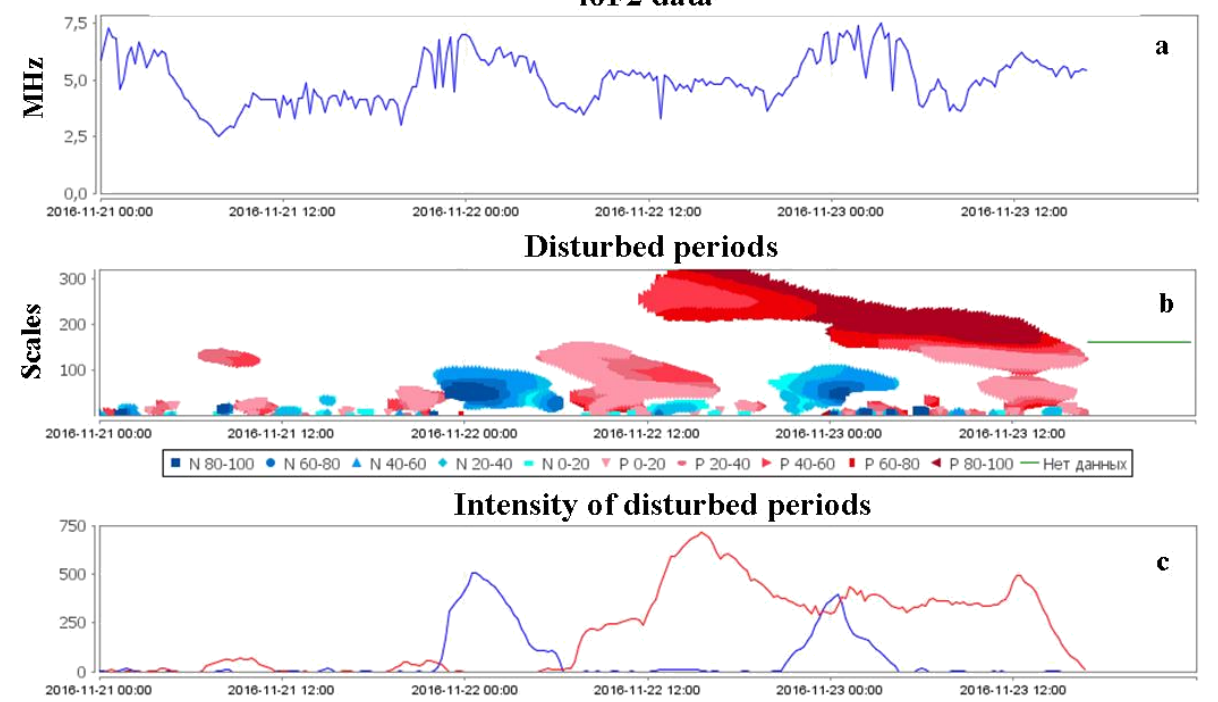

Fig.3. Results of foF2 data processing for the period 2016.11.21 - 2016.11.23. The foF2 data were processed till 17:00 UT on 2016.11.23.

\section{Conclusions}

The paper describes the approaches to ionospheric parameter analysis and anomaly detection. Numerical algorithms were developed for these approaches. They were implemented in software solution block for ionospheric parameter analysis in real-time mode. The software block is a part of «Aurora» geophysical data processing system which is located at the Internet address http://aurorasa.ikir.ru:8580. Software solutions allow us to detect ionospheric anomalies and to assess the dynamics of their change in real-time mode. The investigation was supported by the Russian Scientific Fund Grant (Project No. 14-11- 
00194). The work was carried out by the means of the Common Use Center "North-Eastern Heliogeophysical Center"CKP_558279

\section{References}

1. O.V. Mandrikova, I.S. Solovjev, S.Yu. Khomutov, D.G. Baishev, V.V. Geppener, D.M. Klionskiy, Pattern recognition and image analysis, 26(2016)

2. O.V. Mandrikova, N.V. Fetisova, Y.A. Polozov, I.S. Solovev, M.S. Kupriyanov, Earth, Planets and Space, 67 (2015)

3. O.V. Mandrikova, N.V. Fetisova (Glushkova), R.T. Al-Kasasbeh, D.M. Klionskiy, V.V. Geppener, M.Y. Ilyash, Annals of Geophysics, 58(2015)

4. O. Mandrikova, Yu. Polozov, I. Solovev, N. Fetisova, E3S Web of Conferences, 11 (2016)

5. E.L. Afraimovich, N.P. Perevalova, GPS-monitoring verhnejatmosferyZemli [GPSmonitoring of the Earth's upper atmosphere] (GU NU RVH VSNC SO RAMN, Irkutsk, 2006)

6. N. I. Budko, A.N. Zaitsev, A.T. Karpachev, A.N. Kozlov, B.P. Filippov, Kosmicheskayasredavokrugnas. [The space environment around us] (TROVANT, Troitsk, 2005)

7. A.D. Danilov, Advances in Space Research, 52, 343-366 (2013)

8. G.A. Mansilla, StudiaGeophysica et Geodaetica, 51, 563-574 (2007)

9. G. Box, G. Jenkins, Time series analysis: Forecasting and control (Holden-Day, San Francisco, 1970)

10. O. Mandrikova, N. Fetisova, Yu. Polozov, Machine Learning and Data Analysis (to be published)

11. S. Mallat, A wavelet tour of signal processing (Academic Press, London, 1999)

12. O. Mandrikova, N. Fetisova, Yu. Polozov, Journal of Physics: Conference Series by IOP Publishing (to be published)

13. O. V. Mandrikova, Yu. A. Polozov, I. S. Solovev, N. V. Fetisova(Glushkova), T. L. Zalyaev, M.S. Kupriyanov, A.V. Dmitriev, Pattern Recognition and Image Analysis, 26, 406-418 (2016)

14. Yu. Polozov, N. Fetisova, E3S Web of Conferences, 20 (2017)

15. O. Mandrikova, Yu. Polozov, V. Geppener, Procedia Engineering, 201, 756-766 (2017)

16. O.V. Mandrikova, Yu.A. Polozov, V.V. Bogdanov, E.A. Zhizhikina, A Journal of Software Engineering and Applications, 5, 181-187 (2012) 